\title{
Peripheral and Central Metabolites Affecting Depression, Anxiety, Suicidal Ideation, and Anger in Complex Regional Pain Syndrome Patients Using a Magnetic Resonance Spectroscopy: A Pilot Study
}

\author{
Ye-Ha Jung ${ }^{1}$, Hyeonjin Kim², So Yeon Jeon ${ }^{1}$, Jeong Min Kwon ${ }^{3}$, Won Joon Lee', \\ Joon Hwan Jang ${ }^{4}$, Dasom Lee', Yoonji Lee', and Do-Hyung Kang ${ }^{1,5} \bowtie$ \\ ${ }^{1}$ Department of Neuropsychiatry, Seoul National University Hospital, Seoul, Republic of Korea \\ ${ }^{2}$ Department of Radiology, Seoul National University Hospital, Seoul, Republic of Korea \\ ${ }^{3}$ Department of Experimental Animal Research, Biomedical Research Institute, Seoul National University Hospital, Seoul, Republic of Korea \\ ${ }^{4}$ Department of Medicine, Seoul National University College of Medicine, Seoul, Republic of Korea \\ ${ }^{5}$ Department of Psychiatry, Seoul National University College of Medicine, Seoul, Republic of Korea
}

Objective This study investigated peripheral and central metabolites affecting depression, anxiety, suicidal ideation, and anger in complex regional pain syndrome (CRPS) patients.

Methods Metabolite levels were determined in the right and left thalamus and insula, in 12 CRPS patients using magnetic resonance spectroscopy (MRS).

Results There were positive correlations between valine (Val)/tNAA (N-acetylaspartate+N-acetylaspartylglutamate) and the anxiety, and a negative correlation between glutamine (Gln)/NAA and the depression. There were positive correlations between alanine (Ala)/Gln and the depression and suicidal ideation, between glutamate (Glu)/Gln and the depression and suicidal ideation, between $\mathrm{N}$-acetylaspartylglutamate (NAAG)/Gln and the depression. There was a positive correlation between Ala/NAAG and the trait anger and a negative correlation between creatine (Cr)/N-acetylaspartate (NAA) and the trait anger. There was a negative correlation between $\mathrm{Cr} / \mathrm{Glx}(\mathrm{Glu}+\mathrm{Gln}$ ) and the trait anger. High hemoglobin and alkaline phosphatase were associated with low pain levels, but $\mathrm{CO}_{2}$ and chloride showed positive correlations with pain levels in CRPS patients. Peripheral glucose, $\mathrm{CO}_{2}$ and chloride were associated with depression, anxiety, anger and suicidal ideation.

Conclusion The specific central and peripheral metabolites were associated with psychological disorders including depression, anxiety, suicidal ideation and anger in CRPS patients, showing pathological interactions between a painful body and mind.

Psychiatry Investig 2018;15(9):891-899

Key Words Complex Regional Pain Syndrome, Magnetic Resonance Spectroscopy, Depression, Anxiety, Suicidal ideation, Anger.

\section{INTRODUCTION}

Complex regional pain syndrome type I (CRPS I) refers to severe pain with a complex pathogenesis that is characterized by the involvement of both the peripheral and the central

\section{Received: November 25, 2017 Revised: January 18, 2018} Accepted: June 17, 2018

$\bowtie$ Correspondence: Do-Hyung Kang, MD, PhD

Department of Neuropsychiatry, Seoul National University Hospital, 101 Daehak-ro, Jongno-gu, Seoul 03080, Republic of Korea

Tel: +82-2-2072-0690, Fax: +82-2-747-9063, E-mail: basuare@daum.net

(a) This is an Open Access article distributed under the terms of the Creative Commons Attribution Non-Commercial License (https://creativecommons.org/licenses/bync/4.0) which permits unrestricted non-commercial use, distribution, and reproduction in any medium, provided the original work is properly cited. nervous systems. ${ }^{1,2}$ CRPS is considered to be a neuropathological disorder due to the altered function of the sympathetic nervous system and the presence of maladaptive neuroplasticity, including allodynia and hyperalgesia. ${ }^{2,3}$ More specifically, in vivo proton magnetic resonance spectroscopy ( $\left.{ }^{1} \mathrm{H}-\mathrm{MRS}\right)$ studies have shown that CPRS I patients have lower levels of $\mathrm{N}$-acetylaspartate (NAA) in the dorsolateral prefrontal cortex and higher levels of orbitofrontal myo-inositol (ml). ${ }^{4}$ The structural alterations, neurocognitive dysfunction, ${ }^{5}$ and abnormal changes in specific metabolites present in CRPS patients may also contribute to the impaired recognition of social emotion, ${ }^{6}$ increases in suicidal ideation, ${ }^{7}$ and reduced empathic abilities $^{8}$ observed in CRPS patients. The previous MRS stud- 
ies of other pain syndrome showed similar and different results compared with the previous CRPS study. Chronic pelvic pain patients had significantly lower levels of NAA/creatine (Cr) in the primary motor cortex compared with healthy patients. ${ }^{9}$ And, chronic low back pain (CLBP) patients showed lower NAA levels and higher glutamate (Glu)+glutamine (Gln)/ $\mathrm{Cr}$ and $\mathrm{Glu}+\mathrm{Gln}(\mathrm{Glx}) / \mathrm{mL}$ ratios in the anterior cingulate cortex (ACC) of CLBP patients compared with controls. ${ }^{10}$ CLBP patients showed significant decrease in Glu in patients' ACC and reduced $\mathrm{ml}$ in the ACC and thalamus, ${ }^{11}$ decreased choline (Cho) in the right somatosensory cortex. ${ }^{12}$ Chronic pain patients have statistically significantly higher levels of $\mathrm{Glu} / \mathrm{tCr}$ and $\mathrm{Glx} / \mathrm{tCr}$ and lower levels of NAA/tCr than controls. ${ }^{13}$

CRPS patients exhibit depression, anxiety, somatization, depersonalization, and higher ratings of psychological distress. ${ }^{14-16}$ Additionally, this population is associated with significant risk factors of suicidal ideation such as severity of pain and depressive symptoms, which suggests that psychiatric interventions should be included in the treatment of CRPS. ${ }^{7}$ Similarly, in a model of CRPS, specific neuroplastic changes are associated with anxiety and memory deficits. ${ }^{17}$

MRS has been used to investigate neurometabolic abnormalities in patients with depression and other mental disorders. Depression is associated with lower $\mathrm{mI}$ concentrations in the thalamus and $\mathrm{ACC}^{18}$ and has a negative correlation with the NAA/Cr ratio in the right putamen. ${ }^{19}$ Compared to healthy controls, suicide attempters and depressed patients exhibit lower NAA levels and higher Gln levels. ${ }^{20}$ Additionally, anxiety and depression have a positive relationship with $\mathrm{NAA} / \mathrm{tCr}[\mathrm{Cr}+$ phosphocreatine (PCr)] and Glx/tCr levels in chronic pain patients. ${ }^{21}$ Although the associations of various metabolites with mental disorders in patients with chronic pain have been reported, no studies have investigated both peripheral and central metabolites that affect neuropsychiatric symptoms in CRPS patients. Because the somatosensory system involved in pain perception includes the thalamus, the primary somatosensory cortex, and the insula, ${ }^{22}$ we focused on these regions including the right and left thalamus and insula in CRPS patients. The thalamus is a relay centre subserving both sensory and motor mechanisms as well as pain perception ${ }^{22,23}$ and is involved in the processing of stimuli with emotional content such as depression and anxiety. ${ }^{24,25}$ Thus, we investigated central metabolites which may affect depression, anxiety, and suicidal ideation, focusing on the right and left thalamus in CRPS patients. As the right insula plays a critical role in situations involving anger, ${ }^{26}$ metabolite levels which may affect anger were identified in the insula regions of CRPS patients. The somatosensory system involved in pain perception includes the thalamus, the primary somatosensory cortex, and the insula. ${ }^{27}$ And abnormalities in brain structure and function of CRPS patients were found in prefrontal, motor cortex, cingulate cotex, thalamo-cortical somatosensory maps, and central somatosensory and motor networks. ${ }^{28-31}$ Thus, the aim of the present study was to determine specific peripheral and central metabolites in the right and left thalamus and insula that could affect depression, anxiety, suicidal ideation, and anger in CRPS patients.

\section{METHODS}

\section{Participants}

The present study recruited 12 CRPS patients who met the International Association for the Study of Pain criteria for CRPS type I (Budapest criteria) from Seoul National University Hospital (Seoul, South Korea). All participants underwent a detailed neuropsychiatric assessment at baseline that included a history of accidents, pain symptoms, routine blood analysis, electrocardiogram (ECG), and urine analysis. Subjects with abnormal high-sensitivity C-reactive protein (hsCRP) levels or leukocytosis were excluded from this study.

The inclusion criteria for the CRPS subjects were as follows: a diagnosis of CRPS type I, between 25 and 55 years of age, and not having taken a benzodiazepine or having stopped taking a benzodiazepine medication 2 weeks prior to the study. All eligible patients were approached in the clinic and the project research goals were explained to them. The exclusion criteria were as follows: the presence of a major neuropsychiatric disorder prior to the diagnosis of CRPS, a neurological disease (cerebrovascular disease or brain tumor), history of brain trauma, high hs-CRP levels or leukocytosis, and/or the inability to undergo a magnetic resonance imaging (MRI) scan. From routine blood analyses, we can get the peripheral data. white blood cell, red blood cell, hemoglobin, hematocrit, mean corpuscular volume, mean corpuscular hemoglobin, red blood cell distribution width, platelet, plateletcrit, mean platelet volume, lymphocyte, monocyte, eosinophil, basophil, and absolute neutrophil count were measured using EDTA whole blood. Calcium, phosphorus, glucose, blood urea nitrogen, uric acid, cholesterol, serum total protein, albumin, total bilirubin, alkaline phosphatase, aspartate transaminase, alanine transaminase, creatinine, isotope dilution mass spectrometry-modification of diet in renal disease, $\mathrm{Na}, \mathrm{K}, \mathrm{Cl}$, total $\mathrm{CO}_{2}$, and hs-CRP were measured from blood serum which does not contain white or red blood cells.

The sensory and affective dimensions of current pain were assessed with the short-form McGill Pain Questionnaire (SFMPQ), which includes 11 sensory (MPQ-S) items and four affective (MPQ-A) pain items. The MPQ-S and MPQ-A subscales of the SF-MPQ were included in the analyses. The severity of neuropsychiatric symptoms (depressed mood, anxi- 
ety, suicidal tendencies, and anger) in each CRPS patient was measured with the Beck Depression Beck Depression Inventory (BDI), Beck Anxiety Beck Depression Inventory (BAI), Scale for Suicidal Ideation-Beck (SSI-B), and State-Trait Anger Expression Inventory (STAXI). This study was approved by the Institutional Review Board (IRB No. 1406-099-589) of Seoul National University Hospital (Seoul, South Korea). All data were obtained after written informed consent had been granted by all subjects following a full explanation of the experimental methods.

\section{${ }^{1} \mathbf{H}-M R S$ data acquisition and processing}

All MR data were collected using a 3T human MR scanner with a 16-channel head and neck coil (Siemens Trio system; Siemens Medical Solutions, Erlangen, Germany). For the ${ }^{1} \mathrm{H}$-MRS volume localization, anatomical images were collected using a T2-weighted fast spin echo sequence along the axial (axi), sagittal (sag), and coronal (cor) directions with the following characteristics: repetition time $(\mathrm{TR})=6,090 \mathrm{~ms}$ (axi) and 5,910 $\mathrm{ms}$ (sag and cor), echo time (TE)=89 ms, flip angle $=90^{\circ} / 130^{\circ}$, field of view $(\mathrm{FOV})=220 \times 199 \mathrm{~mm}^{2}$ (axi and cor) and $220 \times 220 \mathrm{~mm}^{2}$ (sag), matrix size $=256 \times 180$, echo train length $=5$, echo spacing $=9.93 \mathrm{~ms}$, receiver bandwidth $(\mathrm{BW})=$ $271 \mathrm{~Hz} /$ pixel, number of slices=30 (no gap), slice thickness= $5 \mathrm{~mm}$, and number of signal averages (NSA) $=128$.

Based on the scout images, four volumes of interest (VOIs) were selected for analysis in each of the 12 subjects: the right and left thalamus $\left(2 \times 2 \times 1.5 \mathrm{~cm}^{3}\right)$ and the right and left insula $\left(2 \times 1.5 \times 2 \mathrm{~cm}^{3}\right)$. The VOIs in the right and left thalamus were placed along the axis of the thalamus to cover most of the volume. After autoshimming over the VOI, ${ }^{1} \mathrm{H}-\mathrm{MRS}$ data were acquired using a point-resolved spectroscopy pulse sequence (PRESS; Bottomley) with the following characteristics: TR/ $\mathrm{TE}=2,000 / 30 \mathrm{~ms}, 2,048$ data points, $\mathrm{BW}=2,500 \mathrm{~Hz}, \mathrm{NSA}=128$, four dummy scans, and four-step phase cycling. The main PRESS sequence was preceded by water and outer-volume suppression modules and the carrier frequency was adjusted by -2.3 ppm from the water resonance to minimize voxel displacement.

The ${ }^{1} \mathrm{H}-\mathrm{MRS}$ data were analyzed with LCModel (Provencher) software (v. 6.3-1J) in the range of 1.0-4.2 ppm; the metabolite content was normalized to that of total creatine $[(\mathrm{tCr})=$ $\mathrm{Cr}+\mathrm{PCr}$ ). Among a variety of MRS-detectable metabolites, we focused on the levels of NAA/tCr, N-acetylaspartylglutamate (NAAG)/tCr, Glu/tCr, Gln/tCr, valine (Val)/tCr, ml/tCr, alanine (Ala)/tCr, glycerophosphocholine (GPC)/tCr, and glutathione (GSH)/tCr. As additional reference markers, we used NAA, NAAG, total NAA (tNAA), Glu, Gln, and Glx. NAAG can be differentiated from NAA in vivo by MRS at $3 \mathrm{~T}^{32} \mathrm{NAA}$, NAAG, Glu, and Gln have been used as additional reference markers. ${ }^{33-36}$ The final data analysis included only metabolites with a Cramer-Rao lower bound (CRLB) $<30 \%$.

\section{Statistical analysis}

The statistical analyses were performed using SPSS Statistics 21.0 (IBM Corp., Armonk, NY, USA). Pearson's correlation analyses were conducted to evaluate the associations between the metabolites and psychological disorders. p-values< 0.05 were considered to indicate statistical significance.

\section{RESULTS}

The present study analyzed the data of 12 patients (nine males and three females) with CRPS who completed all study procedures. The average age was $41.4 \pm 8.6$ (range: 30 to 55 ) years, the average education level was $14.9 \pm 2.3$ years, and the average duration of illness was $8.5 \pm 5.3$ years. There were positive correlations between pain levels and depression, anxiety, and suicidal ideation in the CRPS patients (Table 1), but no correlations between pain levels and anger (Table 1).

\section{Correlations between central neurometabolites and neuropsychiatric assessments in CRPS patients}

The correlations between these neurometabolites and the BDI, BAI, SSI-B, and STAXI scores of the 12 CRPS patients

Table 1. Correlations between pain levels and psychological disorders in CRPS patients

\begin{tabular}{clll}
\hline & MPQ-T & MPQ-S & MPQ-A \\
\hline SSI & & & \\
r & $0.707^{*}$ & $0.854^{* *}$ & 0.419 \\
$\mathrm{p}$ & $0.015^{*}$ & $0.001^{* *}$ & 0.200 \\
BDI & & & \\
r & $0.811^{* *}$ & $0.741^{* *}$ & $0.782^{* *}$ \\
p & $0.002^{* *}$ & $0.009^{* *}$ & $0.004^{* *}$ \\
BAI & & & \\
r & $0.832^{* *}$ & $0.786^{* *}$ & $0.768^{* *}$ \\
p & $0.002^{* *}$ & $0.004^{* *}$ & $0.006^{* *}$ \\
STAXI-S & & & \\
r & 0.163 & 0.210 & 0.081 \\
p & 0.631 & 0.536 & 0.812 \\
STAXI-T & & & \\
r & 0.141 & 0.068 & 0.214 \\
p & 0.679 & 0.843 & 0.528 \\
\hline
\end{tabular}

${ }^{*} \mathrm{p}<0.05,{ }^{* *} \mathrm{p}<0.01$. CRPS: complex regional pain syndrome, BDI: Beck Depression Index, BAI: Beck Anxiety Index, SSI: Scale for Suicidal Ideation, STAXI-S: State-Trait Anger Expression Inventory-State, STAXI-T: State-Trait Anger Expression Inventory-Trait, MPQ-T: McGill Pain Questionnaire-Affective+Sensory, MPQ-A: McGill Pain Questionnaire-Affective, MPQ-S: McGill Pain Questionnaire-Sensory 
were analyzed. For the present study, only strong correlations ( $r>0.800)$ between neurometabolites and neuropsychiatric symptoms were focused. The correlations of depression (BDI), anxiety (BAI) and suicidal ideation (SSI-B) were investigated focused on neurometabolites in the right and left thalamus. In the right thalamus, there were a negative correlation between Gln/NAA and the BDI $\left(r=-0.961, p=0.039, r^{2}=0.924\right)$ (Figure $1 \mathrm{~A}$ ) and a positive correlation between $\mathrm{Val} / \mathrm{tNAA}$ and the BAI ( $\left.r=0.878, p=0.004, r^{2}=0.771\right)$ (Figure 1B). In the left thalamus, there were positive correlations between Ala/Gln and the BDI $\left(\mathrm{r}=0.853, \mathrm{p}=0.015, \mathrm{r}^{2}=0.728\right)$ (Figure 2A) and the SSI $\left(r=0.876, p=0.010, r^{2}=0.767\right)$ (Figure 2A), between Glu/Gln and the BDI $\left(r=0.861, p=0.013, r^{2}=0.741\right)$ (Figure $2 \mathrm{~B})$ and the SSI $\left(\mathrm{r}=0.942, \mathrm{p}=0.005, \mathrm{r}^{2}=0.888\right)$ (Figure $\left.2 \mathrm{~B}\right)$, between NAAG/GIn and the BDI $\left(r=0.811, p=0.027, r^{2}=0.658\right)$ (Figure 2C).

On the other hand, the correlations of trait anger (STAXI-
T) were analyzed with neurometabolites in right and left insula, but not in thalamus, considering that the right insula plays a critical role in situations involving anger. ${ }^{26}$ In the right insula, there were negative correlations between $\mathrm{Cr} / \mathrm{NAA}$ and the STAXI-T ( $r=-0.850, \mathrm{p}=0.015, \mathrm{r}^{2}=0.723$ ) (Figure 3A) and a positive correlation between Ala/NAAG and the STAXI-T $\left(r=0.824, p=0.044, r^{2}=0.679\right)$ (Figure 3B). And there was negative correlation between Cr/Glx and the STAXI-T ( $r=-0.930$, $\mathrm{p}=0.002, \mathrm{r}^{2}=0.865$ ) (Figure $3 \mathrm{C}$ ) in the left insula.

\section{Correlations between peripheral metabolites and pain levels or neuropsychiatric assessments in CRPS patients}

We investigated the relationships between peripheral metabolites and MPQ pain level. Mean corpuscular hemoglobin $(\mathrm{MCH})$ showed a negative correlation with MPQ total pain level $(\mathrm{r}=-0.607, \mathrm{p}=0.036)$ (Figure $4 \mathrm{~A})$. Total $\mathrm{CO}_{2}$ showed a posi-
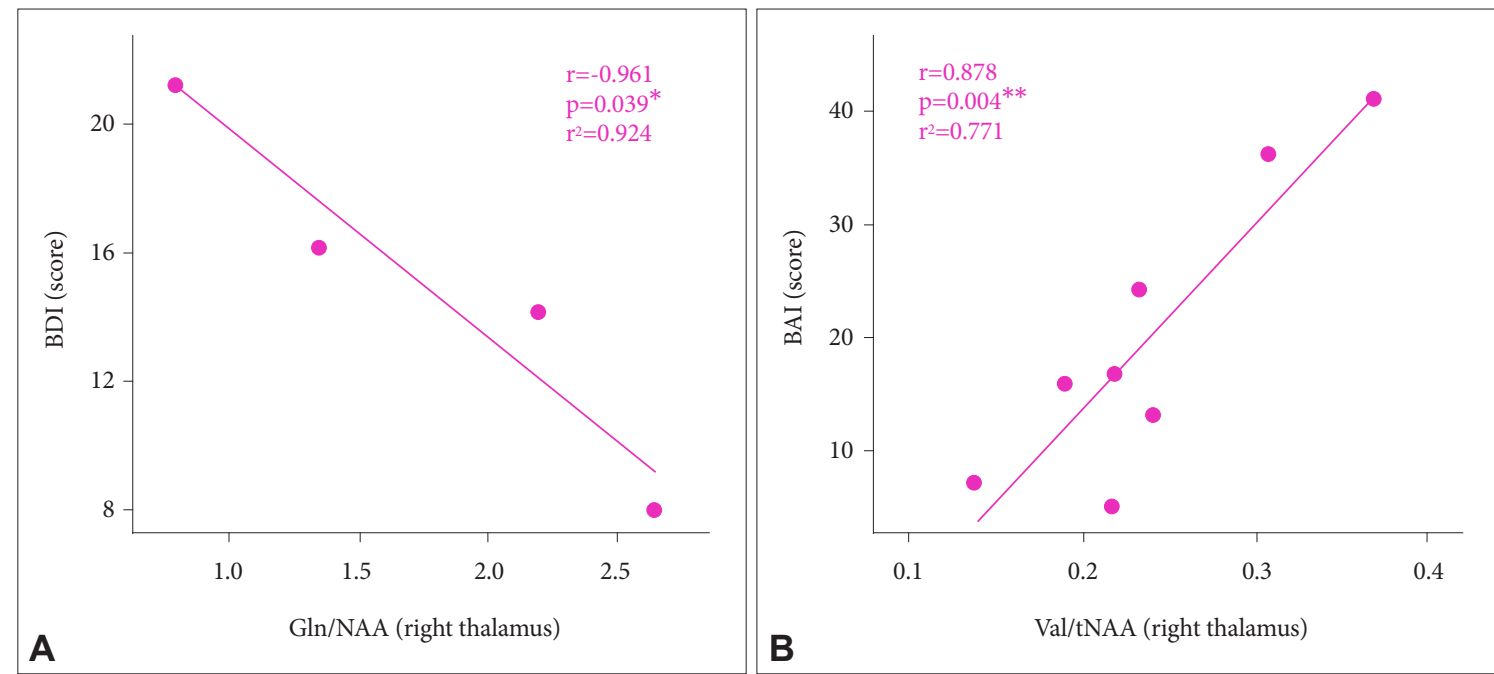

Figure 1. Correlations between depression or anxiety and neurometabolites in right thalamus. (A) $n=4,(B) n=8$. ${ }^{*} p<0.05,{ }^{* *} p<0.01$. BDI: Beck Depression Index, BAI: Beck Anxiety Index, GIn/NAA: glutamine/N-acetylaspartate, Val/tNAA: valine/N-acetylaspartate+N-acetylaspartylglutamate.
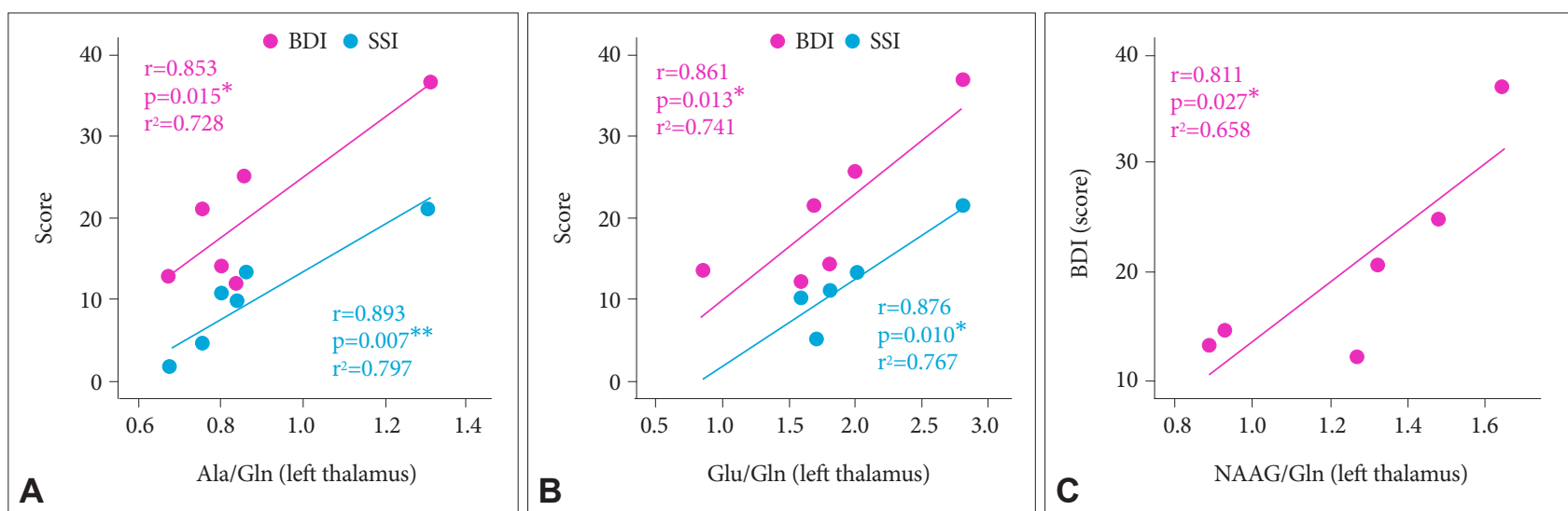

Figure 2. Correlations between depression, anxiety and suicidal ideation and neurometabolites in left thalamus. (A) $n=6$, (B) $n=6$, (C) $n=6$. ${ }^{*} \mathrm{p}<0.05,{ }^{* *} \mathrm{p}<0.01$. BDI: Beck Depression Index, SSI: Scale for Suicidal Ideation, Ala/GIn: alanine/glutamine, Glu/GIn: glutamate/glutamine, NAAG/GIn: N-acetylaspartylglutamate/glutamine. 

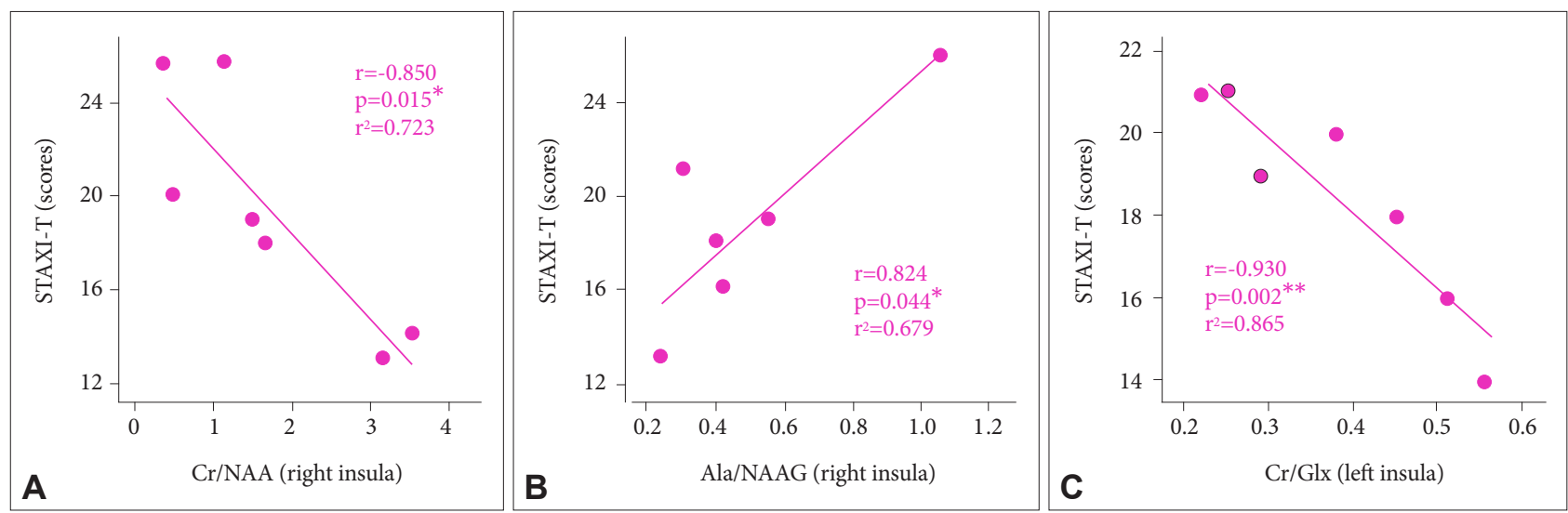

Figure 3. Correlations between trait anger and neurometabolites in right and left insula. (A) $n=7$, (B) $n=6$, (C) $n=7$. STAXI-T: State-Trait Anger Expression Inventory-Trait, ${ }^{*} \mathrm{p}<0.05,{ }^{* *} \mathrm{p}<0.01$. Cr/NAA: creatine/N-acetylaspartate, Ala/NAAG: alanine/ $\mathrm{N}$-acetylaspartylglutamate, $\mathrm{Cr} /$ GIx: creatine/glutamate+glutamine.
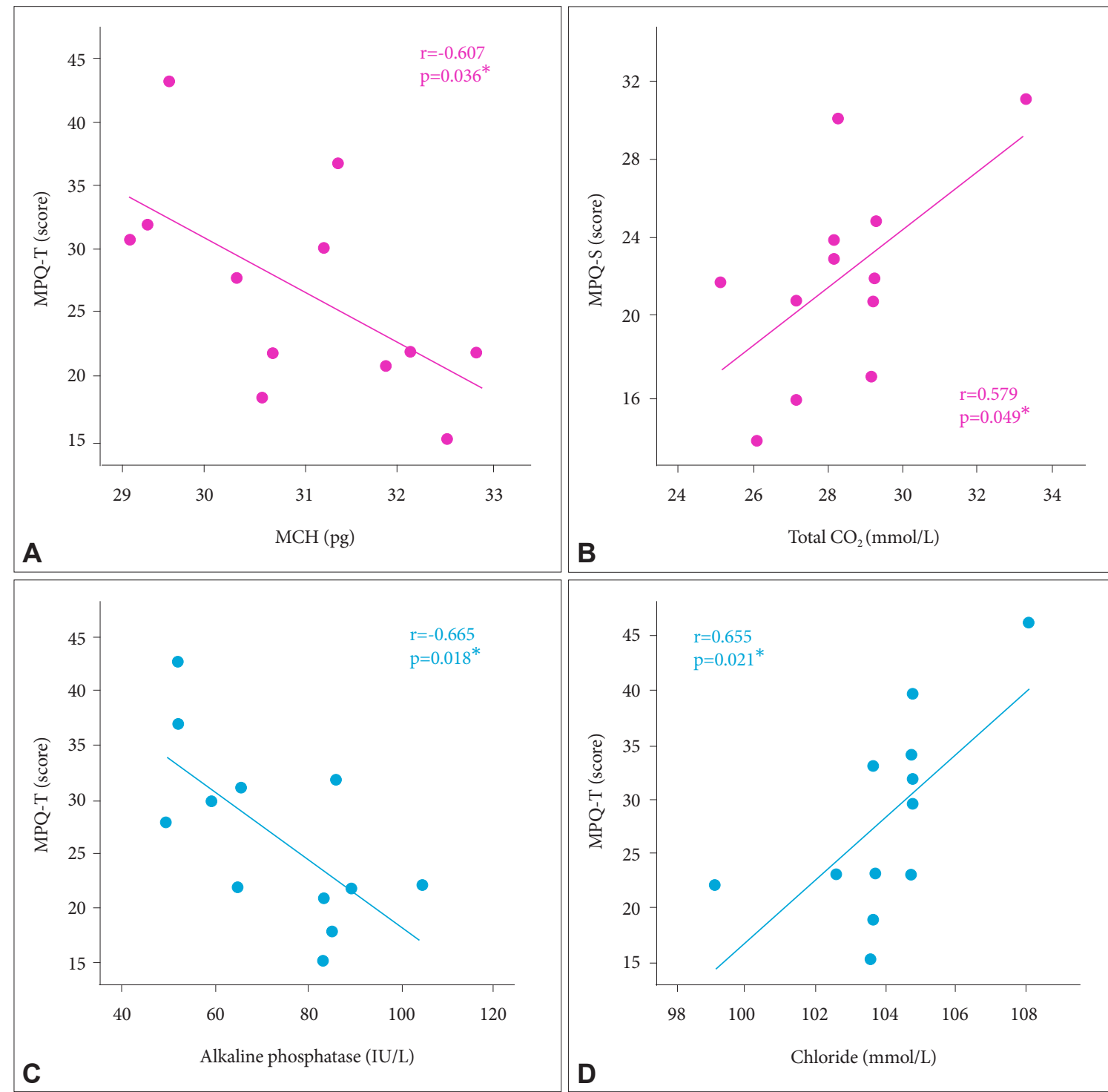

Figure 4. Correlation between peripheral metabolites and MPQ pain levels. (A-D) $n=12 .{ }^{*} p<0.05$. MPQ-T: McGill Pain Questionnaire-Total, MPQ-S: McGill Pain Questionnaire-Sensory, MCH: Mean Corpuscular Hemoglobin. 
tive correlation with MPQ sensory pain $(\mathrm{r}=0.579, \mathrm{p}=0.049)$ (Figure 4B). There was a negative correlation between alkaline phosphatase and MPQ total pain $(\mathrm{r}=-0.665, \mathrm{p}=0.018)$ (Figure $4 \mathrm{C}$ ). There was a positive correlation between chloride and MPQ total pain $(\mathrm{r}=0.655, \mathrm{p}=0.021)$ (Figure $4 \mathrm{D})$. Next, we investigated the associations between peripheral metabolites and neuropsychiatry assessments in CRPS patients. Peripheral glucose showed negative correlations with depression $(r=-0.631, r=0.028)$ (Figure $5 \mathrm{~A})$ and anger $(r=-0.576, p=$ 0.050 ) (Figure $5 \mathrm{~A}$ ). There were positive correlations between total $\mathrm{CO}_{2}$ and depression ( $\mathrm{r}=0.595, \mathrm{p}=0.041$ ) (Figure 5B), anxiety $(\mathrm{r}=0.701, \mathrm{p}=0.011)$ (Figure $5 \mathrm{~B})$ and suicidal ideation $(\mathrm{r}=$ $0.655, \mathrm{p}=0.021$ ) (Figure $5 \mathrm{~B}$ ). Also, there was a positive correlation between chloride and depression $(\mathrm{r}=0.687, \mathrm{p}=0.014)$ (Figure 5C).

\section{DISCUSSION}

CRPS patients have higher levels of depression and anxiety than healthy controls. ${ }^{37}$ Similarly, the present study found that the CRPS patients who had higher pain levels had higher depression and anxiety levels. The present findings also revealed that suicidal ideation was affected by pain levels, which suggests that physical pain increases depression, anxiety, and suicidal ideation in CRPS patients and indicates that there are pathophysiological interactions between a painful body and the mind. The structural alterations and neurocognitive dysfunction observed in previous studies of CRPS patients ${ }^{5}$ and the abnormal changes in specific neurometabolites identified in the present CRPS patients may contribute to the impaired recognition of social emotion, ${ }^{6}$ increases in suicidal ideation, ${ }^{7}$ and depression and anxiety in this population.

Previous MRS studies investigating neurometabolites have associated these levels with psychological variables such as depression and anxiety. For example, lower NAA levels and lower $\mathrm{mI}$ concentrations have been observed in patients with depression, ${ }^{18-20}$ which implies the presence of dysfunctional cell death related to neurons and glial cells. Because our previous studies have shown that CRPS patients already exhibit lower NAA levels and neuroinflammation-dependent elevations in lipid levels, neuronal cell death may affect depressive and anxiety symptoms in CRPS patients.

Although close relationships between neurometabolite levels and physical pain and mental pain were found with similar tendencies in the present study, there were differences between physical pain and mental pain in terms of the reference metabolites. CRPS patients exhibit increases in Ala, GPC, NAAG, $\mathrm{mI}$, and Gln in conjunction with lower levels of NAA relative to $\mathrm{tCr}$ compared to healthy controls in our previous study (will be in press). However, the significant increases in specific metabolites that were related to mental disorders in CRPS patients in the present study were based on different references: NAA and NAAG in the right thalamus and Gln and Glx in the left thalamus. Neuronal cell death may differently affect mental disorders depending on right or left brain regions. Because NAA was reference markers in the right thalamus based on indicators of neuronal cell death, the increases in Val/tNAA over neuronal cell death seem to affect the presentation of psychological symptoms, including anxiety and suicidal ideation, in right thalamus-dependent CRPS patients. On the other hand, left thalamus-dependent CRPS patients showed Gln as reference markers in the significant results. Because an increase in Gln was associated with a decrease in NAA in our previous study (not yet published) and Lip13a is regarded as a marker of necrosis, ${ }^{38}$ the psychological disorders of left region-dependent CRPS patients also seem to be affected by neuronal cell death. The synthesis of Gln from Glu and ammonia in astrocytes is related to ammonia detoxification and protection
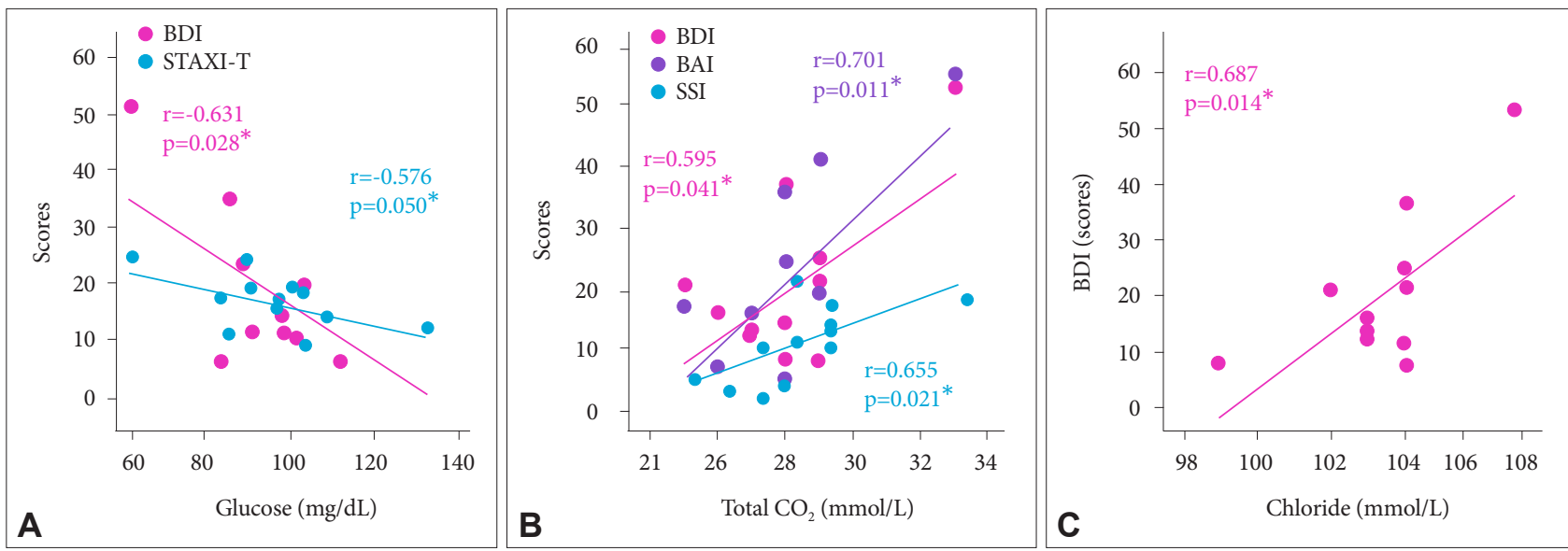

Figure 5. Correlation between peripheral metabolites and neuropsychiatric assessment. (A-C) $n=12$. ${ }^{*}<<0.05$. BDI: Beck Depression Index, BAI: Beck Anxiety Index, SSI: Scale for Suicidal Ideation, STAXI-T: State-Trait Anger Expression Inventory-Trait. 
from excitotoxicity due to excess glutamate. ${ }^{39}$ One of the primary roles of astrocytes is to protect neurons against excitotoxicity by taking up excess ammonia and glutamate, and converting it into glutamine via the enzyme glutamine synthetase. ${ }^{40}$ Also, glutamine synthetase in astrocyte is pivotal in health and disease, clearing ammonia and preventing neurotoxicity in the brain. ${ }^{41}$ Interestingly, the increase in Gln seems to boost the protective mechanisms that underlie decreases in pain levels, depression, and suicidal ideation in CRPS patients. This may not be surprising because Gln is a key pharmaconutrient involved in the body's responses to stress and injury that exerts life-saving interventions. ${ }^{42}$

The right insula plays a critical role in situations involving anger. ${ }^{26}$ In the present study, significant differences in metabolite levels were identified in the insula regions of CRPS patients. More specifically, $\mathrm{Cr}$ in both right and left insula affected trait anger. $\mathrm{Cr}$ can be used as an internal reference for calculating metabolite ratios because its concentrations remain relatively stable. ${ }^{43,44}$ However, in CRPS patients, Cr levels may decrease due to the severe pain and energy deficiencies that result from the use of energy during pain regulation. This may be associated with the observed increases in trait anger given that $\mathrm{Cr}$ is an important organic compound acting as intracellular high-energy phosphate shuttle and in energy storage in muscle and brain tissues. ${ }^{45}$ Additionally, high Ala levels are correlated with high blood pressure and hypertension ${ }^{46,47}$ and thus may also boost trait anger in CRPS patients.

Since glucose is used as an energy source, in addition to the role of energy metabolite $\mathrm{Cr}$, as glucose is also used as an energy source, high levels of serum glucose related to high energy levels may lower trait anger and depression levels in CRPS patients. Recent evidence suggests that glucose dysregulation is associated with increased levels of depression, ${ }^{48}$ and depressive symptoms are associated with lower glucose levels. ${ }^{49}$ Interestingly, considering that people eat chocolate when they feel depressed, high glucose seems to psychologically affect alleviation of depression. Thus, glucose as well as $\mathrm{Cr}$, an energy metabolite seems to play an important role for neuropsychiatric symptoms and psychological well-being in CRPS patients. Given that a rise in the partial pressure of $\mathrm{CO}_{2}$ or a lower $\mathrm{pH}$ will cause offloading of oxygen from hemoglobin known as the Bohr effect, the imbalance and dysfunctional interactions between oxygen and $\mathrm{CO}_{2}$ may affect high levels of pain and psychological disorders such as depression and anxiety. Impaired tissue oxygen saturation in patients with CRPS $1^{50}$ may be associated with pain levels in CRPS. Indeed, hyperbaric oxygen therapy significantly alleviated mechanical allodynia, suggesting the inhibitory role of hyperbaric oxygen therapy in GABAergic neuron apoptosis suppresses ongoing neuropathic pain. ${ }^{51}$ In other words, while high levels of oxy- gen may reduce pain levels, high $\mathrm{CO}_{2}$ levels may increase sensory pain levels, affecting the synergic boost of neuropsychiatric symptoms such as depression, anxiety and suicidal ideation in CRPS patients. Considering that alkaline phosphatase is optimally active at alkaline $\mathrm{pH}$ environments ${ }^{52}$ and is related to a diagnosis of musculoskeletal pain, ${ }^{53}$ high alkaline phosphatase levels in alkaline $\mathrm{pH}$ environments may affect the reduction of pain levels in CRPS patients. Since Cl- flow into or out of the cell plays a crucial role in hyperpolarizing or depolarizing the cells respectively, and neuropathic pain was associated with dysregulation of calcium-activated chloride channels, ${ }^{54}$ high serum chloride levels may contribute to the elevation of pain levels in CRPS. Also, since high chloride levels in serum is associated with high depression levels as well as high pain levels in CRPS patients, chloride dysregulation seems to play an important role in the pathological mechanism of CRPS, affecting higher depression levels.

In the present study, these findings indicate that increases in mental pain may occur due to physical pain and that there is a close relationship between psychological disorders and CRPS. Thus, the present findings suggest that psychiatric interventions should be included in treatment regimens for CRPS to provide relief and enhance mental well-being in addition to alleviating pain. Unlike the internal reference $(\mathrm{tCr})$ for metabolite markers of physical pain, the different metabolites that were identified as internal references played differential but important roles in the mental symptoms associated with pain depending on whether the alterations were in right or left brain regions. This suggests that personalized therapies should be introduced according to the psychological issues of an individual and whether there are right- or left-sided metabolic differences.

This is a pilot study with a small sample size and a preliminary study to be progressed in the following research. Multiple correlations can be accounted for with Bonferroni and other corrections, or by the approach of controlling the false discover rate, but these approaches are not always needed, given that reducing the type I error for null associations increases the type II error for those associations that are not null. ${ }^{55,56}$ We used a rather liberal threshold for significance in correlation analysis, although requires a stricter significance threshold for individual comparisons, so as to compensate for the number of inferences being made. We may miss important findings when we stick to corrections for multiple correlations. Also, as the correlation results were consistent with our previous results and basic theoretical background for pain and psychiatry theory, we think that our findings deserve to be accepted. Because this is a pilot study with a small sample size and a preliminary study, we can confirm these findings and develop gradual progress and breakthrough in the follow- 
ing research. Some people may insist that values with a CRLB $<20 \%$ were viewed as being reliable, but metabolites were considered reliably quantified if the CRLB were $<50 \%{ }^{57}$ Although we used liberal values with a CRLB $<30 \%$ viewed as being reliable, our results were in line with our previous related other findings and theoretical background for pain and MRS study. Also, more liberal and challengeable approach can lead new important findings and make a new way to go breakthrough, given that considering as being reliable values with a CRLB $<30 \%$ can reduce loss of sample numbers which may happen when a CRLB $<20 \%$ viewed as being reliable, if researches based on a CRLB $<30 \%$ report consistently reliable results. Thus, values with a CRLB $<30 \%$ in our study can be viewed as being important to lead progress in human neuroscience and more active researches related to MRS.

In conclusion, to the best of our knowledge, this is the first study to report in vivo MRS data regarding both peripheral and central neurometabolites that affect neuropsychiatric symptoms in CRPS patients. The present study found distinct metabolites that could influence neuropsychiatric symptoms related to CRPS based on NAA-dependent right thalamus and Gln-dependent left thalamus. These results should make an important contribution to the determination and understanding of the complex pathophysiological mechanisms underlying psychological disorders in patients with CRPS. Additionally, these findings should be valuable for comprehending the relationships between peripheral and central metabolic markers of neuropsychiatric symptoms and pain markers of CRPS, and may be useful for the development of effective individual treatments for CRPS that can be based on metabolic differences in the right and left thalamus of these patients.

\section{Acknowledgments}

The authors wish to thank all participants for their valuable time engaging with this research.

This work was supported by Grant No. R-20150902-002176 from the IT R\&D program of MSIP (Ministry of Science, Information and Communication Technology and Future Planning).

\section{REFERENCES}

1. Jänig W, Baron R. Complex regional pain syndrome is a disease of the central nervous system. Clin Auton Res 2002;12:150-164.

2. Bussa M, Mascaro A, Cuffaro L, Rinaldi S. Adult complex regional pain syndrome type I: a narrative review. PM R 2017;9:707-719.

3. Marinus J, Moseley GL, Birklein F, Baron R, Maihöfner C, Kingery WS, et al. Clinical features and pathophysiology of complex regional pain syndrome. Lancet Neurol 2011;10:637-648.

4. Grachev ID, Thomas PS, Ramachandran TS. Decreased levels of Nacetylaspartate in dorsolateral prefrontal cortex in a case of intractable severe sympathetically mediated chronic pain (complex regional pain syndrome, type I). Brain Cogn 2002;49:102-113.

5. Lee DH, Lee KJ, Cho KI, Noh EC, Jang JH, Kim YC, et al. Brain alterations and neurocognitive dysfunction in patients with complex re- gional pain syndrome. J Pain 2015;16:580-586.

6. Shin NY, Kang DH, Jang JH, Park SY, Hwang JY, Kim SN, et al. Impaired recognition of social emotion in patients with complex regional pain syndrome. J Pain 2013;14:1304-1309.

7. Lee DH, Noh EC, Kim YC, Hwang JY, Kim SN, Jang JH, et al. Risk factors for suicidal ideation among patients with complex regional pain syndrome. Psychiatry Investig 2014;11:32-38.

8. Sohn HS, Lee DH, Lee KJ, Noh EC, Choi SH, Jang JH, et al. Impaired empathic abilities among patients with complex regional pain syndrome (Type I). Psychiatry Investig 2016;13:34-42.

9. Simis M, Reidler JS, Duarte Macea D, Moreno Duarte I, Wang X, Lenkinski $\mathrm{R}$, et al. Investigation of central nervous system dysfunction in chronic pelvic pain using magnetic resonance spectroscopy and noninvasive brain stimulation. Pain Pract 2015;15:423-432.

10. Kameda T, Fukui S, Tominaga R, Sekiguchi M, Iwashita N, Ito K, et al. Brain metabolite changes in the anterior cingulate cortex of chronic low back pain patients and correlations between metabolites and psychological state. Clin J Pain 2018;34:657-663.

11. Gussew A, Rzanny R, Güllmar D, Scholle HC, Reichenbach JR. 1HMR spectroscopic detection of metabolic changes in pain processing brain regions in the presence of non-specific chronic low back pain. Neuroimage 2011;54:1315-1323.

12. Sharma NK, McCarson K, Van Dillen L, Lentz A, Khan T, Cirstea CM. Primary somatosensory cortex in chronic low back pain-a H-MRS study. J Pain Res 2011;4:143-150.

13. Ito T, Tanaka-Mizuno S, Iwashita N, Tooyama I, Shiino A, Miura K, et al. Proton magnetic resonance spectroscopy assessment of metabolite status of the anterior cingulate cortex in chronic pain patients and healthy controls. J Pain Res 2017;10:287-293.

14. Rommel O, Willweber-Strumpf A, Wagner P, Surall D, Malin JP, Zenz M. Psychological abnormalities in patients with complex regional pain syndrome (CRPS). Schmerz 2005;19:272-284.

15. Margalit D, Ben Har L, Brill S, Vatine JJ. Complex regional pain syndrome, alexithymia, and psychological distress. J Psychosom Res 2014; 77:273-277.

16. Michal M, Adler J, Reiner I, Wermke A, Ackermann T, Schlereth T, et al. Association of neglect-like symptoms with anxiety, somatization, and depersonalization in complex regional pain syndrome. Pain Med 2017;18:764-772.

17. Tajerian M, Leu D, Zou Y, Sahbaie P, Li W, Khan H, et al. Brain neuroplastic changes accompany anxiety and memory deficits in a model of complex regional pain syndrome. Anesthesiology 2014;121:852-865.

18. Taylor R, Osuch EA, Schaefer B, Rajakumar N, Neufeld RW, Théberge $\mathrm{J}$, et al. Neurometabolic abnormalities in schizophrenia and depression observed with magnetic resonance spectroscopy at $7 \mathrm{~T}$. BJPsych Open 2017;3:6-11.

19. Chagas MH, Tumas V, Pena-Pereira MA, Machado-de-Sousa JP, Carlos Dos Santos A, Sanches RF, et al. Neuroimaging of major depression in Parkinson's disease: cortical thickness, cortical and subcortical volume, and spectroscopy findings. J Psychiatr Res 2017;90:40-45.

20. Jollant F, Near J, Turecki G, Richard-Devantoy S. Spectroscopy markers of suicidal risk and mental pain in depressed patients. Prog Neuropsychopharmacol Biol Psychiatry 2016 [Epub ahead of print].

21. Ito T, Tanaka-Mizuno S, Iwashita N, Tooyama I, Shiino A, Miura K, et al. Proton magnetic resonance spectroscopy assessment of metabolite status of the anterior cingulate cortex in chronic pain patients and healthy controls. J Pain Res 2017;10:287-293.

22. Apkarian AV, Bushnell MC, Treede RD, Zubieta JK. Human brain mechanisms of pain perception and regulation in health and disease. Eur J Pain 2005;9:463-484.

23. Herrero MT, Barcia C, Navarro JM. Functional anatomy of thalamus and basal ganglia. Childs Nerv Syst 2002;18:386-404.

24. Young KA, Holcomb LA, Bonkale WL, Hicks PB, Yazdani U, German DC. 5HTTLPR polymorphism and enlargement of the pulvinar: unlocking the backdoor to the limbic system. Biol Psychiatry 2007;61: 
813-818.

25. Iscan Z, Rakesh G, Rossano S, Yang J, Zhang M, Miller J, et al. A positron emission tomography study of the serotonergic system in relation to anxiety in depression. Eur Neuropsychopharmacol 2017;27:1011-1021.

26. Mazzola V, Arciero G, Fazio L, Lanciano T, Gelao B, Popolizio T, et al. What impact does an angry context have upon us? The effect of anger on functional connectivity of the right insula and superior temporal gyri. Front Behav Neurosci 2016;10:109.

27. Apkarian AV, Bushnell MC, Treede RD, Zubieta JK. Human brain mechanisms of pain perception and regulation in health and disease. Eur J Pain 2005;9:463-484.

28. Pleger B, Draganski B, Schwenkreis P, Lenz M, Nicolas V, Maier C, et al. Complex regional pain syndrome type I affects brain structure in prefrontal and motor cortex. PLoS One 2014;9:e85372.

29. Barad MJ, Ueno T, Younger J, Chatterjee N, Mackey S. Complex regional pain syndrome is associated with structural abnormalities in pain-related regions of the human brain. J Pain 2014;15:197-203.

30. Drummond PD. Sensory disturbances in complex regional pain syndrome: clinical observations, autonomic interactions, and possible mechanisms. Pain Med 2010;11:1257-1266.

31. Schwenkreis P, Maier C, Tegenthoff M. Functional imaging of central nervous system involvement in complex regional pain syndrome. AJNR Am J Neuroradiol 2009;30:1279-1284.

32. Edden RA, Pomper MG, Barker PB. In vivo differentiation of $\mathrm{N}$-acetyl aspartyl glutamate from $\mathrm{N}$-acetyl aspartate at 3 Tesla. Magn Reson Med 2007;57:977-982.

33. Penner J, Rupsingh R, Smith M, Wells JL, Borrie MJ, Bartha R. Increased glutamate in the hippocampus after galantamine treatment for Alzheimer disease. Prog Neuropsychopharmacol Biol Psychiatry 2010; 34:104-110.

34. Fievisohn EM, Sajja VS, Vandevord PJ, Hardy WN. Evaluation of impact-induced traumatic brain injury in the Göttingen Minipig using two input modes. Traffic Inj Prev 2014;15(Suppl 1):S81-S87.

35. Kubo H, Nakataki M, Sumitani S, Iga JI, Numata S, Kameoka N, et al. $1 \mathrm{H}$-magnetic resonance spectroscopy study of glutamate-related abnormality in bipolar disorder. J Affect Disord 2017;208:139-144.

36. Naveed MA, Goyal P, Malhotra A, Liu X, Gupta S, Mangla M, et al. Grading of oligodendroglial tumors of the brain with apparent diffusion coefficient, magnetic resonance spectroscopy, and dynamic susceptibility contrast imaging. Neuroradiol J 2018;31:379-385.

37. Jeon SY, Seo S, Lee JS, Choi SH, Lee DH, Jung YH, et al. [11C]-(R)PK11195 positron emission tomography in patients with complex regional pain syndrome: a pilot study. Medicine (Baltimore) 2017;96: e5735.

38. Zoula S, Hérigault G, Ziegler A. Farion R, Décorps M, Rémy C. Correlation between the occurrence of $1 \mathrm{H}-\mathrm{MRS}$ lipid signal, necrosis and lipid droplets during C6 rat glioma development. NMR Biomed 2003; 16:199-212.

39. Nissim I. Newer aspects of glutamine/glutamate metabolism: the role of acute pH changes. Am J Physiol 1999;277:F493-F497.

40. Suárez I, Bodega G, Fernández B. Glutamine synthetase in brain: effect of ammonia. Neurochem Int 2002;41:123-142.
41. Rose CF, Verkhratsky A, Parpura V. Astrocyte glutamine synthetase: pivotal in health and disease. Biochem Soc Trans 2013;41:1518-1524.

42. Weitzel LR, Wischmeyer PE. Glutamine in critical illness: the time has come, the time is now. Crit Care Clin 2010;26:515-525.

43. Saunders DE, Howe FA, van den Boogaart A, Griffiths JR, Brown MM. Aging of the adult human brain: in vivo quantitation of metabolite content with proton magnetic resonance spectroscopy. J Magn Reson Imaging 1999;9:711-716.

44. Soreni N, Noseworthy MD, Cormier T, Oakden WK, Bells S, Schachar R. Intraindividual variability of striatal (1)H-MRS brain metabolite measurements at 3 T. Magn Reson Imaging 2006;24:187-194.

45. Rackayova V, Cudalbu C, Pouwels PJW, Braissant O. Creatine in the central nervous system: From magnetic resonance spectroscopy to creatine deficiencies. Anal Biochem 2017;529:144-157.

46. Hong X, Wongtongkam N, Ward PR, Xiao S, Wang S, Peng Q, et al. An association of serum ALT with elevated blood pressure in senior adults: a case-control study. Clin Exp Hypertens 2016;38:691-695.

47. Yoo JE, Park HS. Response to: "Relationship between parental hypertension and cardiometabolic risk factors in adolescents: Methodological issues". J Clin Hypertens (Greenwich) 2017;19:825.

48. Nouwen A, Nefs G, Caramlau I, Connock M, Winkley K, Lloyd CE, et al. Prevalence of depression in individuals with impaired glucose metabolism or undiagnosed diabetes: a systematic review and meta-analysis of the European Depression in Diabetes (EDID) Research Consortium. Diabetes Care 2011;34:752-762.

49. Hapunda G, Abubakar A, Pouwer F, van de Vijver F. Depressive symptoms are negatively associated with glucose testing and eating meals on time among individuals with diabetes in Zambia. Diabetes Metab J 2017;41:440-448.

50. Bellingham GA, Smith RS, Morley-Forster P, Murkin JM. Use of near infrared spectroscopy to detect impaired tissue oxygen saturation in patients with complex regional pain syndrome type 1. Can J Anaesth 2014; 61:563-570.

51. Fu H, Li F, Thomas S, Yang Z. Hyperbaric oxygenation alleviates chronic constriction injury (CCI)-induced neuropathic pain and inhibits GABAergic neuron apoptosis in the spinal cord. Scand J Pain 2017;17: 330-338.

52. Ninfa AJ, Ballou DP, Benore M. Biochemistry and Biotechnology. USA: John Wiley \& Sons, 2010, p.229-230.

53. Braunstein NA. Multiple fractures, pain, and severe disability in a patient with adult-onset hypophosphatasia. Bone Rep 2015;4:1-4.

54. Kamaleddin MA. Molecular, biophysical, and pharmacological properties of calcium-activated chloride channels. J Cell Physiol 2018;233: 787-798.

55. Rothman KJ. No adjustments are needed for multiple comparisons. Epidemiology 1990;1:43-46.

56. Saville DJ. Multiple comparison procedures: the practical solution. Am Stat 1990;44:174-180.

57. Bednař́k P, Moheet A, Deelchand DK, Emir UE, Eberly LE, Bareš M, et al. Feasibility and reproducibility of neurochemical profile quantification in the human hippocampus at 3T. NMR Biomed 2015;28:685-693. 